\title{
Níveis de lisina para leitões na fase de creche alimentados com dietas contendo quirera de arroz
}

\author{
Lysine levels in piglet pre-starter diets containing broken rice
}

\begin{abstract}
Niveles de lisina para lechones en fase de preceba con dietas que contienen Arroz partido
\end{abstract}

Víctor L. Hurtado-Nery', Rita TRN Soares ${ }^{2}$

MVZ, MSc, PhD, Universidad de los Llanos. Villavicencio, Colômbia.

Centro de Ciências y Tecnologias Agropecuárias Universidade Estadual do Norte Fluminense, UENF, Campos dos Goytacazes, Brasil. rnobre@uenf.br

Email: vhurtado@unillanos.edu.co

Recibido: Agosto 14 de 2013. $\quad$ Aceptado: Marzo 4 de 2014

\section{Resumo}

Foi conduzido um experimento com o objetivo de avaliar o efeito dos níveis de lisina digestível sobre o desempenho de leitões na fase de creche, alimentados com rações contendo quirera de arroz. Foram utilizados oitenta leitões mestiços de 21 dias de idade e $6.17 \pm 1.02 \mathrm{~kg}$, distribuídos em um delineamento experimental de blocos casualizados, com quatro tratamentos, cinco repetições e quatro leitões por unidade experimental na fase de creche. O experimento foi dividido em dois períodos (pré-inicial e inicial) de 21 dias cada. As rações foram suplementadas com níveis crescentes de lisina sintética para cada período, resultando nos tratamentos com 1.10, 1.25, 1.40 e $1.55 \%$ e $0.77,0.92,1.07$ e $1.22 \%$ de lisina digestível para o primeiro (6.0$13.0 \mathrm{~kg}$ ) e segundo (13.0-28.0 kg) períodos da fase de creche respectivamente. No primeiro período de creche não houve efeito $(P>0.05)$ dos níveis de lisina digestível sobre o consumo de ração e energia nem sobre a conversão alimentar; os níveis de lisina aumentaram de forma linear $(\mathrm{P}<0.05)$ o consumo diário de lisina e de forma quadrática o ganho de peso. No segundo período de creche, houve efeito linear sobre o consumo diário de ração, lisina, energia e conversão alimentar, e efeito quadrático sobre ganho peso diário. Em conclusão os níveis de lisina digestível para máximo ganho de peso foram estimados em 1.355 e $0.972 \%$ para as fases pré-inicial e inicial de creche em leitões com peso de 6.0-13.0 e 13.0-28.0 kg respectivamente.

Palavras chave: alimentos alternativos, desempenho, exigências nutricionais, suínos.

\begin{abstract}
An experiment was made for evaluating the effect of lysine levels on the performance of piglets fed on pre-starter diets containing rice broken and different lysine levels. Eighty, 21-day-old mestizo pigs weighing $6.17 \pm$ $1.02 \mathrm{~kg}$ were used; they were distributed using a randomised complete block experimental design, involving four treatments, five repetitions and four piglets per experimental unit. The trial was divided into two peri-
\end{abstract}


ods (I pre-initial and II initial), each lasting 21 days. The diets were supplemented with increasing levels of synthetic lysine for each period, resulting in treatments having $1.10 \%, 1.25 \%, 1.40 \%$ and $1.55 \%$ digestible lysine for the first pre-starter phase $(6.0-13.0 \mathrm{~kg})$ and $0.77 \%, 0.92 \%, 1.07 \%$ and $1.22 \%$ for the second one (13.0-28.0 kg). Digestible lysine levels for the pre-initial pre-starter period had no effect ( $p>0.05)$ on feed and energy consumption, or feed conversion ratio (FCR); lysine levels increased linearly $(p<0.05)$ regarding daily digestible lysine consumption and quadratically for weight gain. There was a linear effect on the consumption of feed, lysine, energy and FCR during the second pre-starter period and a quadratic effect on average daily weight gain. Digestible lysine levels for maximum weight gain were thus estimated as being $1.355 \%$ for piglets weighing 6.0 to $13.0 \mathrm{~kg}$ during pre-initial pre-starter phase and $0.972 \%$ for piglets during initial pre-starter phase weighing 13.0 to $28.0 \mathrm{~kg}$.

Key words: Alternative feed, performance, nutritional requirement, piglet.

\section{Resumen}

Se llevó a cabo un experimento para evaluar el efecto de los niveles de lisina en el rendimiento de los lechones en fase de preceba alimentados con dietas con arroz partido y diferentes niveles de lisina. Se utilizaron ochenta cerdos mestizos de 21 días de edad y $6.17 \pm 1.02 \mathrm{~kg}$, distribuidos en un diseño experimental de bloques completos al azar, con cuatro tratamientos, cinco repeticiones y cuatro lechones por unidad experimental. El ensayo fue divido en dos períodos (I pre-inicial y II inicial) de 21 días cada uno. Las dietas fueron suplementadas con niveles crecientes de lisina sintética para cada período, lo que resultó en los tratamientos con $1,10,1.25,1.40$ y $1.55 \%$ y $0.77,0.92,1.07$ y $1.22 \%$ de lisina digerible para el primer $(6.0 \mathrm{a} 13.0 \mathrm{~kg})$ y segundo (13.0-28.0 kg) períodos de la fase de preceba, respectivamente. Para el periodo pre-inicial de preceba no hubo efectos ( $P>0.05$ ) de los niveles de lisina digestible sobre el consumo de alimento y de energía, ni sobre la conversión alimenticia; los niveles de lisina aumentaron linealmente $(\mathrm{P}<0.05)$ el consumo diario lisina digestible y en forma cuadrática la ganancia de peso. En el segundo período de preceba, hubo efecto lineal sobre el consumo de alimento, de lisina, de energía y la conversión alimenticia y efecto cuadrático sobre la ganancia diaria promedio. En conclusión, los niveles de lisina digestible para la máxima ganancia de peso se estimaron en 1.355 y $0.972 \%$ para cerdos en fase pre inicial e inicial de preceba y con peso de 6.0 a 13.0 y de 13.0 a $28.0 \mathrm{~kg}$, respectivamente.

Palabras clave: Alimentos alternativos, rendimiento, requerimientos nutricionales, cerdos.

\section{Introdução}

A lisina é o primeiro aminoácido limitante na nutrição de suínos, e é utilizado como referência para as estimativas das exigências nutricionais dos outros aminoácidos, as quais são estabelecidas utilizando-se a metodologia de dose-resposta com suínos de diferentes idades. Na determinação das exigências dos aminoácidos tem sido aplicado o conceito de proteína ideal com o propósito de fornecer ao suíno um balanço de aminoácidos que supra suas exigências sem excesso nem deficiência e, considerando como base a digestibilidade verdadeira e total dos mesmos (Moreira et al. 2004; Oliveira et al. 2006). Segundo Sakomura e Rostagno (2007), os critérios para estabelecer as exigências de aminoácidos nos suínos são o ganho de peso diário, a conversão alimentar, excreção de ureia, e o nível de aminoácidos no plasma.

A utilização de alimentos alternativos na alimentação de suínos visa reduzir os custos de produção sem pre- judicar o desempenho dos animais, nem a qualidade do produto que chega ao consumidor. A quirera é um subproduto da indústria de arroz com $14 \%$ de grãos quebrados (Limberger et al., 2008), que não é destinado para o consumo humano; além disto, a quirera tem valores nutricionais próximos ao milho (Rostagno et al., 2011), com alta digestibilidade (Ebert et al. 2005), e tem preço inferior ao milho, constituindo-se em uma matéria prima alternativa com alto potencial de uso na alimentação de suínos.

Fatores como a idade, peso e estresse do desmame, baixo consumo de alimento, composição da dieta, imaturidade digestiva e o ambiente, influenciam o crescimento dos suínos em fase de creche (Ribeiro, et al., 2006). O leitão após a desmama deve ser alimentado com rações de alta digestibilidade, atendendo as exigências nutricionais para essa fase de crescimento. A digestibilidade verdadeira da lisina contida na quirera de arroz é de 88.28\% (Apolônio et al. 2003). 
Para as fases de creche em diferentes faixas de peso, valores discrepantes de exigências de lisina digestível têm sido estimados. Assim, Nunes et al. (2008) estimaram a exigência de lisina digestível em $1.46 \%$ para máximo ganho de peso de leitões de 6-15 kg; entretanto para suínos de 15-30 kg foi estimada a exigência de lisina digestível em 1.10\% (Oliveira et al. 2006); e Zangeronimo et al (2007) recomendam $1.05 \%$ de exigência de lisina digestível verdadeira para suínos de 9 aos, $25 \mathrm{~kg}$ de peso vivo.

Considerando que as exigências de nutrientes têm sido estabelecidas com dietas baseadas em milho, é recomendado realizar as correções necessárias para a correta formulação da dieta, quando for necessário utilizar outros ingredientes. Dessa forma, o objetivo deste trabalho foi avaliar o efeito dos níveis de lisina digestível sobre o desempenho de leitões na fase de creche alimentados com rações contendo quirera de arroz em substituição total ao milho.

\section{Material e métodos}

O trabalho foi conduzido na Unidade de Apoio à Pesquisa do Laboratório de Zootecnia e Nutrição Animal da Universidade Estadual do Norte Fluminense, localizada no Município de Campos dos Goytacazes, Rio de Janeiro, Brasil, a $21^{\circ} 45^{\prime} 14^{\prime \prime}$ de latitude sul, $41^{\circ} 19^{\prime}$ $26^{\prime \prime}$ de longitude oeste e $14 \mathrm{msnm}$.

Foram utilizados 80 leitões mestiços Landrace $\mathrm{x}$ Large White $x$ Pietrain, desmamados aos 21 dias de idade, com $6.17 \pm 1.02 \mathrm{~kg}$ de peso, distribuídos em um delineamento experimental de blocos ao acaso; com quatro tratamentos, cinco repetições e quatro animais por unidade experimental. O critério aplicado para a formação dos blocos foi o peso inicial dos leitões.

Os animais foram alojados em um galpão de alvenaria, coberto com telhas de amianto, em baias dotadas de comedouros convencionais e bebedouros automáticos tipo chupeta. No piso das baias foi colocada maravaIha, e a 40 centímetros de altura uma lâmpada, com o propósito de fornecer calor suplementar aos leitões. A temperatura do galpão foi monitorada diariamente com termômetros de máxima e mínima, instalados a altura de $50 \mathrm{~cm}$.

O ensaio foi realizado durante 42 dias na fase de creche com dois períodos, pré-inicial $(6-13 \mathrm{~kg}$ de peso) e inicial (13 -28 kg de peso) de 21 dias cada. As dietas experimentais (Tabela 1) foram fareladas e formuladas para atender as exigências nutricionais, exceto para lisina. Para as faixas de peso de 7.5 a 15 e, 15 a $30 \mathrm{~kg}$ recomendadas por Rostagno et al. (2005).
Tabela 1. Composição centesimal das rações experimentais para leitões na fase de creche

\begin{tabular}{|c|c|c|}
\hline \multirow{2}{*}{ Ingredientes } & \multicolumn{2}{|c|}{ Peso dos leitões, kg } \\
\hline & $6.0-13-0$ & $13.0-28.0$ \\
\hline Farelo de soja & 32.930 & 23.820 \\
\hline Quirera de arroz & 53.833 & 72.042 \\
\hline Fosfato Bicálcico & 1.781 & 1.742 \\
\hline Calcário calcítico & 0.600 & 0.542 \\
\hline Leite em pó & 5.000 & - \\
\hline Açúcar & 2.000 & - \\
\hline Óleo de soja & 1.900 & - \\
\hline Vitaminas $^{1}$ & 0.400 & 0.400 \\
\hline Minerais ${ }^{2}$ & 0.240 & 0.240 \\
\hline Sal & 0.485 & 0.455 \\
\hline L - Lisina $\mathrm{HCl}(98.5 \%)$ & 0.000 & 0.000 \\
\hline Metionina & 0.050 & 0.025 \\
\hline Treonina & 0.171 & 0.124 \\
\hline Inerte & 0.610 & 0.614 \\
\hline \multicolumn{3}{|l|}{ Composição calculada, \% } \\
\hline Proteína bruta & 21.130 & 16.67 \\
\hline EM (kcal / kg) & 3325 & 3290 \\
\hline Fósforo disponível & 0.450 & 0.400 \\
\hline Cálcio & 0.825 & 0.720 \\
\hline Lisina digestível & 1.100 & 0.770 \\
\hline Metionina digestível & 0.372 & 0.278 \\
\hline Treonina digestível & 0.828 & 0.624 \\
\hline Sódio & 0.230 & 0.200 \\
\hline Fibra crua & 2.078 & 1.600 \\
\hline
\end{tabular}

Níveis de garantia por kg do produto: Biotina $16.56 \mathrm{mg}$; Vit. E 10.500 mg;Piridoxina 700 mg; Vit. K3 2.800 mg; Colina 126 g; Niacina 13.650 mg; Acido Pantotênico 7.350 mg; Vit. A 2.800 UI; Tiamina 700 mg; Vit. B12 11.550 mcg; Vit. D3, 1.050 UI; Acido Fólico $420 \mathrm{mg}$; Riboflavina $2.100 \mathrm{mg}$; Antioxidante $1.500 \mathrm{mg} .{ }^{2}$ Níveis de garantia por kg do produto: $\mathrm{Fe}, 45.000 \mathrm{mg}$; $\mathrm{Cu}, 37.000$ mg; Mn, 25.000 mg; Co, 300 mg; I, 800 mg; Se, 120 mg.

As dietas foram suplementadas com níveis crescentes (0.000; $0.191 ; 0.382$ e $0.573 \%$ ) de lisina sintética $\mathrm{HCl}$ $(78.5 \%)$, resultando em tratamentos com 1.10, 1.25, $1.40,1.55 \%$, e $0.77,0.92,1.07,1.22 \%$ de lisina digestível para os períodos correspondentes. Os ingredientes utilizados na dieta foram analisados segundo os procedimentos recomendados por Silva e Queiroz (2004) para proteína bruta pelo método Kjeldahle, em bomba calorimétrica Parr para energia bruta.

As dietas experimentais e a água foram fornecidas à vontade. Para estimar as variáveis de desempenho os suínos foram pesados no inicio e final de cada período experimental; do mesmo modo, as sobras foram pesadas semanalmente para estabelecer o consumo de ração, pela diferença entre o fornecimento e as sobras nos comedouros.

As variáveis analisadas foram consumo de ração diário médio (CDR), ganho de peso diário médio (GDP), conversão alimentar (CA), consumo de lisina diário $(\mathrm{CDL})$ e consumo diário médio de energia (CDE). Os dados obtidos foram processados no programa SAEG, e submetidos à análise de variância e de regressão polinomial. 


\section{Resultados e discussão}

Durante a fase experimental foi constatada a temperatura máxima y mínima de $26.7 \pm 2.1$ y $18.3 \pm 2.0^{\circ} \mathrm{C}$., respectivamente; valores considerados na zona de conforto térmico (Ferreira, 2005), adequada para os leitões na fase de creche de $6-28 \mathrm{~kg}$ de peso e com 21 a 63 dias de idade. Os dados de desempenho de suínos nas fases de creche são apresentados nas Tabelas 2 e 3.

Os níveis de lisina não influenciaram o CDR nem a CA $(\mathrm{P}>0.05)$ de leitões na fase de creche dos 6 aos, $13 \mathrm{~kg}$ de peso vivo (tabela 2). Observou-se efeito linear $(\mathrm{P}<0.05)$ sobre $\mathrm{CDL}$ e quadrático sobre o ganho de peso. O ganho de peso diário foi incrementado até o nível de 1,40\% de lisina digestível. Pela derivação da equação de regressão $-0.4111 x^{2}+1.1141 x-0.3926$; $\mathrm{R}^{2}=0.895$, o nível para máximo ganho de peso foi estimado em $1.355 \%$ de lisina digestível (Figura 1).

O valor estimado é menor ao recomendado de 1,38\% de lisina digestível por Moreira et al. (2005) para leitoas dos 6 aos, $16 \mathrm{~kg}$, com alto potencial para deposição de carne magra na carcaça, e superior á exigência preconizada por Rostagno et al. (2011) de 1.33\% para leitões machos castrados, fêmeas e machos inteiros de alto potencial genético na fase pré-inicial na faixa 9.3$15 \mathrm{~kg}$ de peso vivo, com 33-42 dias de idade.

O ganho de peso diário obtido neste experimento foi superior ao constatado por Nepomuceno (2010), utilizando diferentes níveis de quirera de arroz na dieta para suínos na fase de creche de 21-42 dias de idade. Do mesmo modo, o ganho diário foi inferior ao constatado por Neto et al., (2009) de 441.1 - 457.4 gramas alimentando suínos com diferentes níveis de lisina y de energia em fase inicial de creche dos 6.5 aos $11.5 \mathrm{~kg}$ de peso.

A resposta no desempenho aos níveis de lisina digestível difere dos resultados obtidos por Neto et al. (2004) que verificaram efeito linear da concentração de lisina sobre o ganho diário de peso em leitões de 5.5 até $11 \mathrm{~kg}$, trabalhando com níveis de lisina total entre 1.30 e $1.60 \%$, sugerindo uma maior eficiência na utilização da lisina contida na dieta pelos leitões nesta fase.

Por outro lado, Nunes et al. (2008) não encontram influência dos níveis de lisina no consumo de ração, porém, constataram efeito linear sobre o ganho de peso

Tabela 2. Desempenho de suínos em fase de creche dos 6.0 aos $13.0 \mathrm{~kg}$ alimentados com rações contendo quirera de arroz e diferentes níveis de lisina.

\begin{tabular}{|c|c|c|c|c|c|}
\hline & \multicolumn{5}{|c|}{ Níveis de lisina digestível, \% } \\
\hline & 1.10 & 1.25 & 1.40 & 1.55 & CV \\
\hline Duração experimento, dias & 21 & 21 & 21 & 21 & \\
\hline Ganho de peso diario ${ }^{1}, \mathrm{~kg}$ & 0.337 & 0.353 & 0.366 & 0.345 & 14.48 \\
\hline Consumo diário de ração ${ }^{2}, \mathrm{~kg}$ & 0.528 & 0.504 & 0.516 & 0.500 & 6.5 \\
\hline Conversão alimentar $^{2}$ & 1.612 & 1.510 & 1.449 & 1.537 & 6.97 \\
\hline Consumo diário de lisina ${ }^{3}, \mathrm{~g}$ & 5.81 & 6.30 & 7.22 & 7.75 & 6.8 \\
\hline Consumo diário de energia ${ }^{2}, \mathrm{kcal}$ & 1754 & 1673 & 1713 & 1660 & 6,5 \\
\hline
\end{tabular}

${ }^{1}$ Efeito quadrático $y=-0.4111 x^{2}+1.1141 x-0.3926 ; R^{2}=0.895$

${ }^{2} \mathrm{NS},(\mathrm{P}>0.05)$

${ }^{3}$ Efeito linear $(\mathrm{P}<0.01) ; \hat{\mathrm{Y}}=1.56797+3.34204 \mathrm{x}$

Tabela 3. Desempenho de suínos em fase de creche dos $13-0$ aos $28.0 \mathrm{~kg}$ alimentados com rações contendo quirera de arroz e diferentes níveis de lisina.

\begin{tabular}{|c|c|c|c|c|c|}
\hline & \multicolumn{5}{|c|}{ Níveis de lisina digestível, \% } \\
\hline & 0.77 & 0.92 & 1.07 & 1.22 & CV \\
\hline Duração experimento, dias & 21 & 21 & 21 & 21 & \\
\hline Ganho de peso diario', kg & 0.645 & 0.688 & 0.702 & 0.682 & 7.04 \\
\hline Consumo diário de ração² ${ }^{2}$ kg & 1.224 & 1.217 & 1.183 & 1.168 & 3.28 \\
\hline Conversão alimentar ${ }^{3}$ & 1.981 & 1.785 & 1.744 & 1.754 & 11.94 \\
\hline Consumo diário de lisina ${ }^{4}, g$ & 9.42 & 11.19 & 12.66 & 14.25 & 3.48 \\
\hline Consumo diário de energia ${ }^{5}, \mathrm{kcal}$ & 3954 & 3932 & 3822 & 3773 & 3.28 \\
\hline
\end{tabular}

1 Efeito quadrático $(\mathrm{P}=0.04), \mathrm{R}^{2}=1.0, \mathrm{Y}=-0.071145+1.4655 \mathrm{x}-0.695291 \mathrm{x}^{2}$

${ }^{2}$ Efeito linear $(P<0.01), R^{2}=0.94, Y=1.4447+0.0467778 x$

${ }^{3}$ Efeito linear $(P=0.05), R^{2}=0.70, Y=4.50655-5.05599 x$

${ }^{4}$ Efeito linear $(P=0.00), R^{2}=1.0, Y=-0.637346+14.657 x$

${ }^{5}$ Efeito linear $(\mathrm{P}<0.01), \mathrm{R}^{2}=0.94, \mathrm{Y}=4.01964-0.294289 \mathrm{x}$ 


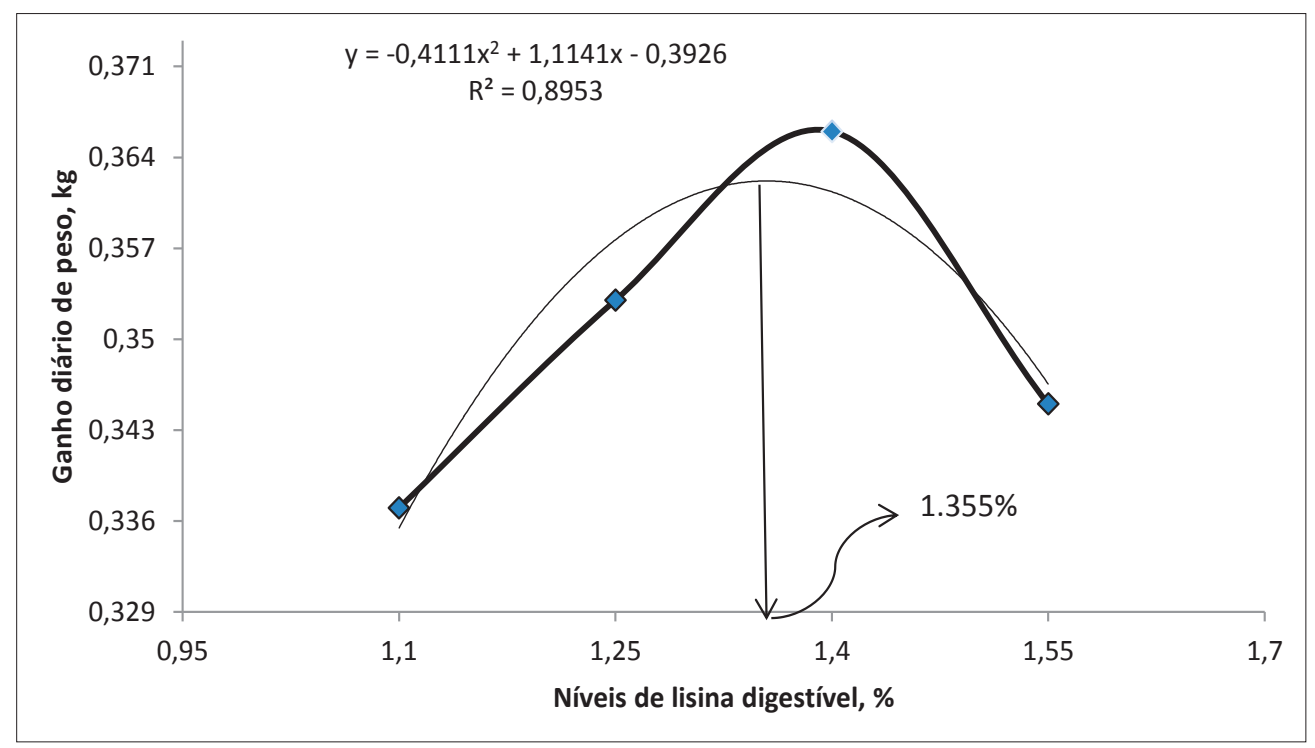

Figura 1. Efeito dos níveis de lisina digestível sobre o ganho de peso para suínos na fase de creche dos 6 aos $13 \mathrm{~kg}$.

e consumo de lisina, sugerindo $1.46 \%$ como exigência de lisina digestível para suínos dos 6 aos 15 kg de peso.

Os altos valores de exigência de lisina digestível para a faixa de peso em estudo pode-se explicar pelo potencial genético dos animais utilizados e pela melhor utilização da lisina para aumentar a deposição de carne e acelerar o crescimento dos animais.

Para a fase dos 13 aos $28 \mathrm{~kg}$ (Tabela 3) observou-se efeito quadrático $(\mathrm{P}<0.05)$ dos níveis de lisina digestível so- bre ganho de peso diário, e efeito linear negativo sobre consumo diário de ração $(\mathrm{P}<0.05)$, de energia metabolizável e a conversão alimentar $(\mathrm{P}<0.05)$ e efeito linear positivo sobre o consumo diário de lisina $(\mathrm{P}<0.01)$.

Para máximo ganho de peso diário o nível de lisina digestível pelo modelo quadrático foi estimado em $1.05 \%$ (Figura 2), e pelo modelo Linear Response Plateau, LRP, como $0.95 \%$. Para esta faixa de peso, pela interseção das curvas (Figura 2) dos dois modelos estimou-se a exigência de lisina digestível em $0.972 \%$,

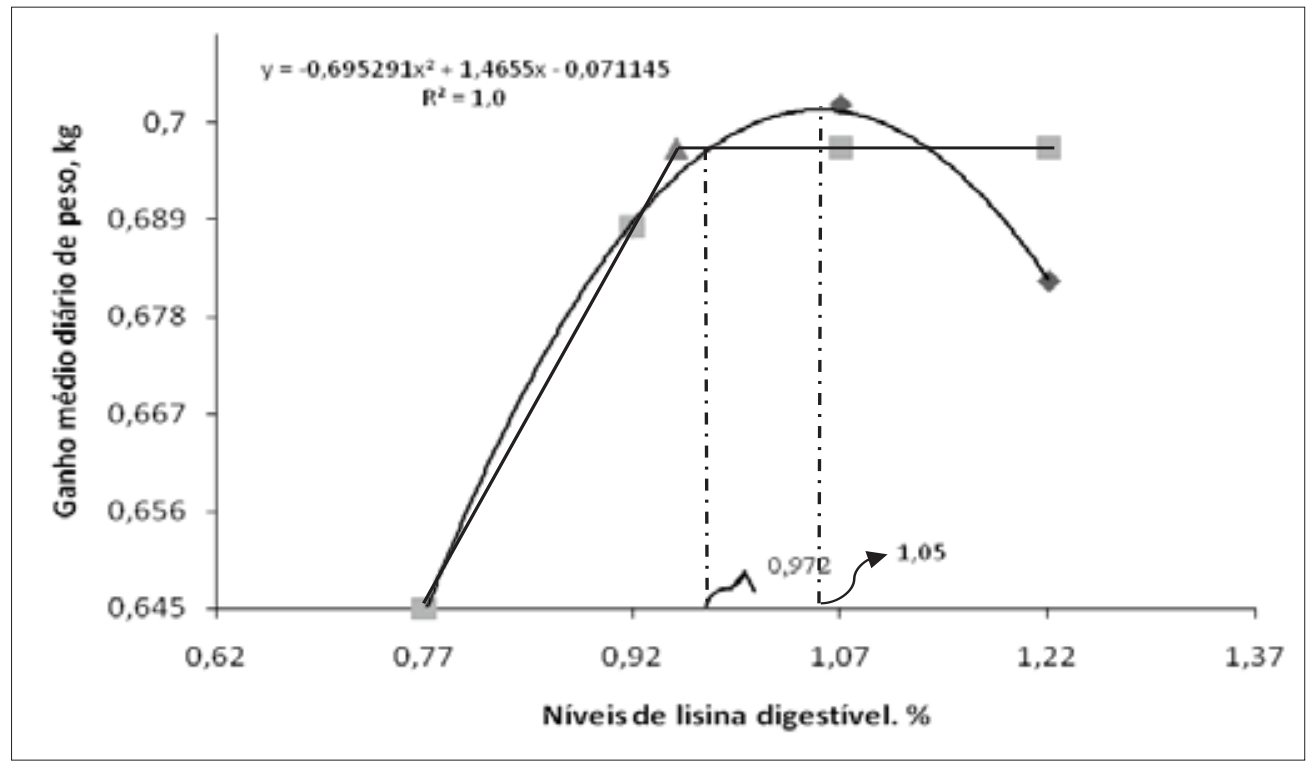

Figura 2. Efeito dos níveis de lisina digestível sobre o ganho de peso para suínos na fase de creche dos 13 aos $28 \mathrm{~kg}$. 
o que corresponde a um consumo de 14 gramas/dia de lisina digestível. Este valor é menor que a exigência preconizada por Rostagno et al. (2011) que é de $1.037 \%$ para suínos machos castrados de alto potencial genético com desempenho médio na faixa de peso 15-30 kg; e menor aos valores estimados por Rossoni et al. (2009) de $1.08 \%$ para fêmeas selecionadas para deposição de carne magra.

O ganho de peso diário obtido neste experimento é superior ao obtido por Neto et al. (2009) de 0.511 $0.556 \mathrm{~kg}$ com suínos na fase de creche dos 11.5 aos $21.9 \mathrm{~kg}$, utilizando diferentes níveis de lisina e ao preconizado NRC (2012) de 0.693 kg para suínos na faixa de $11-25 \mathrm{~kg}$ de peso.

O consumo diário de lisina é inferior ao recomendado por Rostagno et al., (2011) para suínos de 15-30 kg, de 12.02 gramas/dia, e, próximo ao estimado por Moretto et al (2000) que determinaram 15 gramas/dia para suínos inteiros da raça Landrace dos 15 aos $30 \mathrm{~kg}$ de peso. Os mesmos autores relacionaram a variação dos resultados entre os trabalhos com fatores como genótipo dos animais, sistema de alimentação, ambiente e perfil aminoacídico da ração basal, entre outros.

Por outro lado, Oliveira et al. (2006) constataram $1.10 \%$ de exigência de lisina para suínos machos castrados de alto potencial genético para deposição de carne magra na carcaça de suínos dos 15 aos 30 $\mathrm{kg}$, explicando que a resposta de ganho de peso ao nível de lisina varia de acordo com o potencial de crescimento muscular dos animais.

Os resultados de desempenho de suínos na fase de creche nas faixas de peso 6-13 e 13-28 kg, e alimentados com rações que contendo quirera de arroz com diferentes níveis de lisina, são próximos aos resultados onde utilizaram rações baseadas em milho como principal constituinte da dieta (Moreira et al., 2005; Nunes et al., 2008; Zangeronimo et al., 2007).

O aumento linear do consumo de lisina se explica pela concentração crescente nas rações referentes aos tratamentos e, o efeito quadrático sobre o consumo de energia pelo fato das rações serem isocalóricas, uma vez que o consumo de energia esta relacionado ao consumo de ração pelos animais, o que sugere que os suínos podem tolerar alguns excessos de lisina sem apresentar variações no consumo de ração nem no ganho de peso diário. A alta exigência de lisina digestível pode ser explicada pelo potencial genético dos animais, que são selecionados para deposição de carne magra na carcaça, exigindo maiores quantidades de lisina na ração para atender este propósito.

\section{Conclusões}

Os níveis estimados de 1.335 e $0.972 \%$ de lisina digestível na dieta permitem obter o máximo ganho de peso diário em suínos alimentados com rações contendo quirera de arroz, nas fases de creche pré-inicial de 6.0-13.0 kg e inicial de $13-28 \mathrm{~kg}$ de peso vivo, respectivamente.

\section{Agradecimentos}

Á Fundação de Amparo à Pesquisa do Estado do Rio de Janeiro, Faperj, pelo apoio financeiro para a realização desta pesquisa.

\section{Referencias}

Apolônio LR, Donzele JL, Oliveira RFM, Souza AVC, Silva FCO. Bünzen, S. Digestibilidade ileal de aminoácidos de alguns alimentos, determinada pela técnica da cânula T simples com suínos. Rev bras zootec. 2003; 32(3): 605-614.

Ebert AR, Ribeiro AML, Kessler AM. Desempenho e digestibilidade de leitões recém desmamados recebendo grãos de arroz, milho ou farinha de trigo escura. Arch latinoam prod anim. 2005;13(2): 43-50.

Ferreira RA. 2005. Maior produção com melhor ambiente para aves, suínos e bovinos. Ed. Aprenda Fácil, Viçosa, p.371.

Limberger VM, Silva LP, Emanuelli T, Comarela, CG, Patias LD. Modificação química e física do amido de quirera de arroz para aproveitamento na indústria de alimentos. Química Nova. 2008; 31 (1): 84-88.

Moreira HFV, Fontes DO, Silva FCO, Silva MA, Fontes FAPV, Gomes FFE, Rossoni MC. Níveis de lisina para leitoas dos 6 aos $16 \mathrm{~kg}$ com alto potencial para deposição de carne magra na carcaça. Rev bras zootec. 2005; 34(4):1210-1216.

Moreira I, Kutschenko M, Furlan AC, Murakami AE, Martins EN, Scapinello C. Exigência de lisina para suínos em crescimento e terminação, alimentados com rações de baixo teor de proteína, formuladas de acordo com o conceito de proteína ideal. Acta sci anim sci, 2004; 26(4):537-542.

Moretto V, Donzele JL, Oliveira RFM. Fontes DO. Níveis dieteticos de lisina para suínos da raca Landrace dos 15 aos 30 kg. Rev bras zootec. 2000; 29(3):803-809.

National Research Council-NRC. 2012. Nutrient requirements of swine. 11. ed. Washington, D.C.: National Academy of Sciences. 400p.

Nepomuceno RC. 2010. Inclusão da quirera de arroz em rações de suínos na fase de creche. Tese de MSc. Universidade Federal de Ceara, Fortaleza, 52 p.

Neto MAT, Berto DA, Alburquerque R, Schammass EA. Relação lisina digestível e energia metabolizável para leitões em fase pré-inicial de creche. Rev bras zootec. 2009; 38(7):1291-1300. 
Neto MAT, Petelincar IM, Berto DA, Schammass EA, Bisinoto KS, Caldara, FR. Níveis de lisina para leitões na Fase inicial I do crescimento pós-desmame: Composição corporal aos 11,9 e 19,0 kg. Rev bras zootec. 2004; 33(6) sup 1:1777-1789.

Nunes CGV, Oliveira RFM, Donzele JL, Siqueira JC, Pereira AA, Silva BA. Níveis de lisina digestível para leitões dos 6 aos 15 kg. Rev bras zootec. 2008; 37(1):84-88.

Oliveira ALS, Donzele JL, Oliveira RFM, Abreu MLT, Ferreira AS, Silva FCO, Haese D. Exigência de lisina digestível para suínos machos castrados de alto potencial genético para deposição de carne magra na carcaça dos 15 aos 30 kg. Rev bras zootec. 2006; 35(6):2338-2343.

Ribeiro AML, Pedrozzo SA, Kessler AM. Threonine : lysine relations on weaning piglets performance and metabolismo, Rev bras agrociênc. 2006; 12(2):205-210.

Rossoni MC; Donzele JL; Silva FCO; Oliveira RFM; Abreu MLT; Santos FA; Kill JL. Pereira CMC. Exigência de lisina digestível de fêmeas suínas selecionadas para de- posição de carne magra, na carcaça dos 15 aos 30 kg1. Rev bras saúde prod Anim, 2009;10(3):586-595.

Rostagno HS, Albino LFT, Donzele JL, Gomes PC, Oliveira RF, Lopes DC, Ferreira AS, Barreto SLT. 2005. Tabelas Brasileiras para aves e suínos. Composição de alimentos e exigências nutricionais. ed. Viçosa:UFV, $2^{a}$ ed. p.186.

Rostagno HS, Albino LFT, Donzele JL, Gomes PC, Oliveira RF, Lopes DC, Ferreira AS, Barreto SLT, Euclides RF. 2011. Tabelas Brasileiras para aves e suínos. Composição de alimentos e exigências nutricionais. ed. Viçosa:UFV, $3^{\underline{a}}$ ed. 252 pp.

Sakomura NK, Rostagno HS. 2007. Métodos de pesquisa em nutrição de monogástricos. Jaboticabal: UNESP, p. 283.

Zangeronimo MG, Fialho ET, Murgas LDS, Fonseca RT, Rodriguez PB. Desempenho e excreção de nitrogênio de leitões dos 9 aos $25 \mathrm{~kg}$ alimentados com dietas com diferentes níveis de lisina digestível e proteína bruta. Rev bras zootec. 2007; 36(5):1382-1387. 